\title{
RUIN PROBLEMS UNDER IBNR DYNAMICS
}

\author{
Julien Trufin ${ }^{*}$ Hansjörg Albrecher †and Michel Denuit $\ddagger$
}

\begin{abstract}
In this paper, we consider a discrete-time risk process allowing for delay in claim settlement, which introduces a certain type of dependence in the process. From martingale theory, an expression for the ultimate ruin probability is obtained, and Lundberg-type inequalities are derived. The impact of delay in claim settlement is then investigated. To this end, a convex order comparison of the aggregate claim amounts is performed with the corresponding non-delayed risk model, and numerical simulations are carried out with Belgian market data.
\end{abstract}

Key words and phrases: discrete-time risk process, convex order, IBNR claims, large deviations, martingale, ultimate ruin probability.

\section{Introduction}

In classical risk theory, the stochastic process describing the aggregate claim amounts filed against the insurance company is assumed to have independent increments. In a variety of situations this independence assumption appears rather unrealistic. This is for instance the case when considering delay in claim reporting or settlement. The financial result of a given calendar year then depends on the result of one or several preceding years.

In the literature, some risk models allowing for delay in claim settlement have been discussed. Waters and Papatriandafylou (1985) considered a discrete-time risk model with possible delay in claim settlements and derived upper bounds on the ultimate ruin probability. Boogaert and Haezendonck (1989) studied the mathematical properties of the liability process with settlement delay within the framework of an economical environment.

*Julien Trufin, Institut de Statistique, Biostatistique et Sciences Actuarielles (ISBA), Université Catholique de Louvain, Louvain-la-Neuve, Belgium, e-mail: julien.trufin@uclouvain.be

${ }^{\dagger}$ Hansjörg Albrecher, Faculty of Business and Economics, University of Lausanne, CH-1015 Lausanne, Switzerland, email: hansjoerg.albrecher@unil.ch

${ }_{\ddagger}^{\ddagger}$ Michel Denuit, Institut de Statistique, Biostatistique et Sciences Actuarielles (ISBA), Université Catholique de Louvain, Louvain-la-Neuve, Belgium, e-mail: michel.denuit@uclouvain.be 
Klüppelberg and Mikosch (1995) proposed to describe delayed claims in terms of Poisson shot noise processes. Ruin estimates for this model have been studied by Brémaud (2000), who used large deviations theory to give Cramér-Lundberg type approximations for the ultimate ruin probability, see also Macci and Torrisi (2004) and Macci et al. (2005). More generally, Ganesh and Torrisi (2006) considered shot-noise Cox processes and obtained ruin probability estimates for heavy-tailed claims.

A risk process combining delay in claim settlement and reserve-dependent premium rate can be found in Ganesh et al. (2007), where asymptotic estimates of the ultimate ruin probability as well as a most likely path leading to ruin are derived. Yuen et al. (2005) consider a compound Poisson risk model in which each claim induces an additional delayed claim (called by-claim).

In this paper, we study ruin problems in the presence of delay in claim settlement with a discrete-time risk model. Our approach is quite different from the one in Waters and Papatriandafylou (1985). In Waters and Papatriandafylou (1985), the claims development referring to a calendar year operates in a stochastic way, while the ruin event occurs when the reserves for IBNR (incurred but not reported) claims that the insurer needs exceed the amount of available cash. Here, on the other hand, we use a setting that incorporates the Chain-Ladder development factors, widely used in practice to account for IBNR claims. In particular, we assume that the payment of each annual claim is spread over several years according to the development factors (where the spread is applied in a deterministic fashion). This leads to a moving average model for the annual claims that describes in a certain way the dependence of annual claims caused through delayed claims and that allows for a transparent comparison of ruin probabilities with and without IBNR dynamics.

Besides the actuarial setting adopted in this paper, the model used here has a number of other potential applications to non-insurance situations. For instance, it may describe a warranty management process where some of the items sold may become defective and must be repaired at producer's cost. The producer may then set a yearly provision intended to cover the cost of future repairs to items sold during that year (the insurance premium). Some of these items become defective during the year they are sold, one year afterwards, two years afterwards, and so on until the end of the warranty period, causing random costs (the insurance claims). If in addition to the yearly provisions there is also some additional reserve for the future repairing costs, the analogue of the ruin event in this context is then the situation that the money reserved for repairing is not sufficient at some point in time.

In a medical context, the number of individuals affected by a disease may be diagnosed in the year of its occurrence, one year afterwards, two years afterwards etc. A health provider may 
then set provisions in order to cover the future treatment costs and again the above model may be appropriate to study whether the provisions are sufficient. More generally, the model may be applicable whenever there are random events (or shocks) that have quantitative (e.g. monetary) adverse effects on several of the subsequent time units and these random events are counterbalanced by deterministic quantitative protection cushions.

The paper is organized as follows. In Section 2, we describe the risk model used in this paper for the insurer's surplus. In Section 3, we use the martingale method to obtain an explicit expression for the ultimate ruin probability for light-tailed claims. A generalization to the case of a surplus dynamics with a constant interest rate is illustrated in the appendix. Subsequently Lundberg-type inequalities are derived. In Section 4, asymptotic estimates for ultimate ruin probabilities are determined in terms of the Lundberg coefficient, and an insensitivity property of our model is highlighted. This property is also proved for the heavy-tailed case. Finally, in Section 5, the impact of this kind of IBNR dynamics on ruin probability is investigated both for the light-tailed and heavy-tailed claim case. A comparison is made with the associated non-delayed risk model. We establish, for instance, a convex order relation between the IBNR aggregate claims process and the non-delayed aggregate claims process. Numerical illustrations are then performed with Belgian market data.

\section{The Model}

Let $n$ be the number of years needed to settle all the claims related to a given accident year. Let $X_{i}$ be the amount paid in calendar year $i$ for the claims occurring in the same year. The $X_{i}$ s are assumed to be independent with common distribution function $F_{X}$, and we denote as $X$ a generic random variable with distribution function $F_{X}$. The annual claim amount of the company is assumed to be given by

$$
Y_{i}=X_{i}+X_{i-1}\left(\lambda_{1}-1\right)+\sum_{j=i-n+1}^{i-2} X_{j}\left(\lambda_{i-j}-1\right) \prod_{l=1}^{i-j-1} \lambda_{l}
$$

where the $\lambda_{i}$ s are called development factors and are larger than 1 . In practice, these factors are associated to the Chain-Ladder method for loss reserving. So, for our model, the surplus of the insurance company at time $k$ obeys the dynamics

$$
U_{k}=u+k c-\sum_{i=1}^{k} Y_{i}
$$

with $Y_{i}$ described by (2.1), where $u$ is the initial amount of capital and $c$ is the annual premium income. 
Remark 1: Notice that $Y_{i}$ can alternatively be written as

$$
Y_{i}=\beta_{1} S_{i}+\beta_{2} S_{i-1}+\cdots+\beta_{n} S_{i-n+1}
$$

where $S_{i}$ is the ultimate cost for the claims originated in year $i$ and $\beta_{j}$ is the proportion of $S_{i}$ paid after $j$ years. Some practitioners prefer the representation (2.3) rather than (2.1). Assuming that the $S_{i}$ s are independent and identically distributed like $S$, the equivalence between (2.1) and (2.3) results from $S_{i}=X_{i} \prod_{j=1}^{n-1} \lambda_{j}, \beta_{1}=\frac{1}{\prod_{j=1}^{n-1} \lambda_{j}}$ and

$$
\begin{cases}\beta_{2}=\frac{\lambda_{1}-1}{\lambda_{1}} & \text { if } n=2 \\ \beta_{j}=\frac{\left(\lambda_{j-1}-1\right) \prod_{l=1}^{j-2} \lambda_{l}}{\prod_{l=1}^{n-1} \lambda_{l}} \quad j=2, \ldots, n & \text { if } n>2 .\end{cases}
$$

Remark 2: All the results obtained in this paper can be extended without difficulty to the case of link factors depending on accident years, keeping the $X_{i}$ s independent and identically distributed and imposing the same distribution function for the ultimate costs relating to each accident year, i.e. $\prod_{j=1}^{n-1} \lambda_{j}^{(i)}=d$ for all $i$ and for a certain value of $d$, where the $\lambda_{j}^{(i)}$ 's, $j=1, \ldots, n-1$ are now the link ratios for accident year $i$. Such an extension is in line with practice, as speed in settlement may vary over time. In this context, the equation (2.1) then becomes

$$
Y_{i}=X_{i}+X_{i-1}\left(\lambda_{1}^{(i-1)}-1\right)+\sum_{j=i-n+1}^{i-2} X_{j}\left(\lambda_{i-j}^{(j)}-1\right) \prod_{l=1}^{i-j-1} \lambda_{l}^{(j)}
$$

\section{Ultimate ruin probability for light-tailed claims}

\subsection{Expression for the ultimate ruin probability}

Define

$$
\left\{\begin{array}{l}
d_{1}=\lambda_{1}-1 \\
d_{i}=\left(\lambda_{i}-1\right) \prod_{l=1}^{i-1} \lambda_{l} \quad i=2, \ldots, n-1,
\end{array}\right.
$$

and $d=1+d_{1}+\cdots+d_{n-1}$. Clearly, $d=\prod_{j=1}^{n-1} \lambda_{j}$. Let us denote the ultimate ruin probability as

$$
\begin{aligned}
& \psi\left(u, x_{0}, x_{-1}, \ldots, x_{-n+2}\right)= \\
& \quad \operatorname{Pr}\left[U_{k}<0 \text { for some } k \mid U_{0}=u, X_{0}=x_{0}, X_{-1}=x_{-1}, \ldots, X_{-n+2}=x_{-n+2}\right] .
\end{aligned}
$$

We are now in a position to establish the following result. Its proof is inspired by Gerber (1982).

In the following, for a random variable $Z$, we will denote by $\mu_{Z}$ and $\sigma_{Z}^{2}$ its mean and variance, respectively. Furthermore, its distribution function will be denoted by $F_{Z}=1-\overline{F_{Z}}$. 
Theorem 3.1. Assume that there exists a constant $\rho>0$ such that

$$
e^{-\frac{\rho}{d} c} \mathbb{E}\left[e^{\rho X}\right]=1
$$

Then we have

$$
\psi\left(u, x_{0}, x_{-1}, \ldots, x_{-n+2}\right) \leq \frac{e^{-\frac{\rho}{d} \hat{u}}}{\mathbb{E}\left[e^{-\frac{\rho}{d} \hat{U}_{T}} \mid T<\infty\right]},
$$

where $\hat{U}_{k}=U_{k}-\sum_{l=0}^{n-2} \alpha_{l} X_{k-l}\left(\right.$ with $\left.\alpha_{l}=d_{l+1}+\cdots+d_{n-1}\right), \hat{u}=\hat{U}_{0}$ and $T=\inf \left\{k \mid U_{k}<0\right\}$. Proof. Let $\Phi_{k}$ be the sigma-algebra generated by $\left\{X_{i}, i=1,2, \ldots, k\right\}$. We then have

$$
\mathbb{E}\left[e^{-\frac{\rho}{d} \hat{U}_{k}} \mid \Phi_{k-1}\right]=\mathbb{E}\left[e^{-\frac{\rho}{d}\left(U_{k}-\sum_{l=0}^{n-2} \alpha_{l} X_{k-l}\right)} \mid \Phi_{k-1}\right]=\mathbb{E}\left[e^{-\frac{\rho}{d}\left(U_{k-1}+c-Y_{k}-\sum_{l=0}^{n-2} \alpha_{l} X_{k-l}\right)} \mid \Phi_{k-1}\right] .
$$

Now

$$
Y_{k}+\sum_{l=0}^{n-2} \alpha_{l} X_{k-l}=X_{k}+\sum_{j=1}^{n-1} d_{j} X_{k-j}+\sum_{l=0}^{n-2} \alpha_{l} X_{k-l}=d X_{k}+\sum_{l=0}^{n-2} \alpha_{l} X_{k-1-l}
$$

Hence,

$$
\begin{aligned}
\mathbb{E}\left[e^{-\frac{\rho}{d} \hat{U}_{k}} \mid \Phi_{k-1}\right] & =\mathbb{E}\left[e^{-\frac{\rho}{d}\left(U_{k-1}+c-d X_{k}-\sum_{l=0}^{n-2} \alpha_{l} X_{k-1-l}\right)} \mid \Phi_{k-1}\right] \\
& =e^{-\frac{\rho}{d} c} \mathbb{E}\left[e^{\rho X}\right] \mathbb{E}\left[e^{-\frac{\rho}{d}\left(U_{k-1}-\sum_{l=0}^{n-2} \alpha_{l} X_{k-1-l}\right)} \mid \Phi_{k-1}\right] \\
& =e^{-\frac{\rho}{d} \hat{U}_{k-1}} \text { by assumption. }
\end{aligned}
$$

This shows that the stochastic process $\left\{e^{-\frac{\rho}{d} \hat{U}_{k}}, k \geq 1\right\}$ is a martingale.

Let $m$ be a positive integer. By the Optional Stopping Theorem (considering the stopping time $T \wedge m=\min \{T, m\})$, we get

$$
\begin{aligned}
e^{-\frac{\rho}{d} \hat{U}_{0}} & =\mathbb{E}\left[e^{-\frac{\rho}{d} \hat{U}_{T \wedge m}}\right] \\
& =\mathbb{E}\left[e^{-\frac{\rho}{d} \hat{U}_{T}} I_{T \leq m}\right]+\mathbb{E}\left[e^{-\frac{\rho}{d} \hat{U}_{m}} I_{T>m}\right] . \\
& \geq \mathbb{E}\left[e^{-\frac{\rho}{d} \hat{U}_{T}} \mid T \leq m\right] \operatorname{Pr}[T \leq m]
\end{aligned}
$$

Letting $m \rightarrow \infty$ gives

$$
e^{-\frac{\rho}{d} \hat{U}_{0}} \geq \mathbb{E}\left[e^{-\frac{\rho}{d} \hat{U}_{T}} \mid T<\infty\right] \psi\left(u, x_{0}, \ldots, x_{-n+2}\right),
$$

which completes the proof.

This result can easily be generalized to the case of a constant compound interest rate $i \geq 0$, as is shown in the appendix. 
Corollary 3.2. Under the assumptions of Theorem 3.1, if there exists a positive constant $b$ such that $\operatorname{Pr}[X<b]=1$, then we have the equality

$$
\psi\left(u, x_{0}, x_{-1}, \ldots, x_{-n+2}\right)=\frac{e^{-\frac{\rho}{d} \hat{u}}}{\mathbb{E}\left[e^{-\frac{\rho}{d} \hat{U}_{T}} \mid T<\infty\right]} .
$$

Proof. Let $\alpha=\sum_{l=0}^{n-2} \alpha_{l}$. Since $\operatorname{Pr}[X<b]=1$, we have

$$
e^{-\frac{\rho}{d} \hat{U}_{m}} I_{T>m}<e^{\frac{\rho}{d} b \alpha}
$$

Furthermore, the random variables $e^{-\frac{\rho}{d} \hat{U}_{m}} I_{T>m}$ converge to zero pointwise for $m \rightarrow \infty$. Hence, we get

$$
\lim _{m \rightarrow \infty} \mathbb{E}\left[e^{-\frac{\rho}{d} \hat{U}_{m}} I_{T>m}\right]=0
$$

It is well-known that if the security loading $\eta=\frac{c}{d \mu_{X}}-1$ is positive and if $\mathbb{E}\left[e^{r X}\right]$ exists for all $r$ in the neighborhood of the origin and is steep (which in particular implies light-tailed claims), the Lundberg equation $e^{-r \mu_{X}(1+\eta)} \mathbb{E}\left[e^{r X}\right]=1$ admits only one positive solution.

\subsection{Lundberg-type inequalities for the ultimate ruin probability}

Lundberg-type inequalities can be derived from Theorem 3.1. Note that from $\hat{U}_{T}<0$ we have $\mathbb{E}\left[e^{-\frac{\rho}{d} \hat{U}_{T}} \mid T<\infty\right]>1$. Hence, we get the next Proposition.

Proposition 3.1. Under the assumptions of Theorem 3.1, we have

$$
\psi\left(u, x_{0}, \ldots, x_{-n+2}\right) \leq e^{-\frac{\rho}{d} \hat{u}}
$$

Now, let us define $\theta$ as the largest positive integer such that $\operatorname{Pr}[T \geq \theta]=1$. Of course, when there is no constant $b$ such that $\operatorname{Pr}[X<b]=1$, we always have $\theta=1$. Then, the following Proposition, based on (3.4), improves the upper bound (3.6).

Proposition 3.2. Under the assumptions of Theorem 3.1, we have

$$
\psi\left(u, x_{0}, \ldots, x_{-n+2}\right) \leq e^{-\frac{\rho}{d}\left(\bar{u}+\alpha \mu_{X}\right)} .
$$

where

$$
\bar{u}= \begin{cases}u-\sum_{l=0}^{n-3} \alpha_{l} \tilde{x}_{-l}-\alpha_{n-2} x_{-n+2} & \text { if } \theta \leq n-2, \\ \hat{u} & \text { otherwise, }\end{cases}
$$

with $\tilde{x}_{-l}=\max \left(\mu_{X}, x_{-l}\right)$. 
Proof. As $e^{-\frac{\rho}{d} U_{T}}>1$, we have

$$
\psi\left(u, x_{0}, \ldots, x_{-n+2}\right) \leq \frac{e^{-\frac{\rho}{d} \hat{u}}}{\mathbb{E}\left[e^{\frac{\rho}{d} \sum_{l=0}^{n-2} \alpha_{l} X_{T-l}} \mid T<\infty\right]} \leq \frac{e^{-\frac{\rho}{d} \hat{u}}}{e^{\frac{\rho}{d} \sum_{l=0}^{n-2} \alpha_{l} \mathbb{E}\left[X_{T-l} \mid T<\infty\right]}}
$$

by Jensen's inequality. Since $\mathbb{E}\left[X_{i} \mid T<\infty\right] \geq \min \left(x_{\theta-n+2}, \ldots, x_{0}\right)$ for $\theta-n+2 \leq i \leq 0$, we have $\mathbb{E}\left[X_{T-l} \mid T<\infty\right] \geq \gamma$ for $l=0, \ldots, n-2$, where

$$
\gamma=\left\{\begin{array}{ll}
\min \left(x_{\theta-n+2}, \ldots, x_{0}, \mu_{X}\right) & \text { if } \theta \leq n-2 \\
\mu_{X} & \text { otherwise }
\end{array} .\right.
$$

Hence

$$
\psi\left(u, x_{0}, \ldots, x_{-n+2}\right) \leq e^{-\frac{\rho}{d}(\hat{u}+\alpha \gamma)},
$$

where we recognize (3.7) for $\theta>n-2$. If $\theta \leq n-2$, it suffices to see that $\psi\left(u, x_{0}, \ldots, x_{-n+2}\right) \leq$ $\psi\left(u, \tilde{x}_{0}, \ldots, \tilde{x}_{-n+3}, x_{-n+2}\right)$.

Note that for $\theta \leq n-2$, we have from (3.7) and (3.9)

$$
\psi\left(u, x_{0}, \ldots, x_{-n+2}\right) \leq \min \left(e^{-\frac{\rho}{d}(\hat{u}+\alpha \gamma)}, e^{-\frac{\rho}{d}\left(\bar{u}+\alpha \mu_{X}\right)}\right) .
$$

But since $\bar{u} \leq \hat{u}$ and $\gamma \leq \mu_{X}$, we always have $e^{-\frac{\rho}{d}(\hat{u}+\alpha \gamma)} \geq e^{-\frac{\rho}{d}\left(\bar{u}+\alpha \mu_{X}\right)}$.

Clearly, the upper bound $e^{-\frac{\rho}{d}\left(\bar{u}+\alpha \mu_{X}\right)}$ is tighter than $e^{-\frac{\rho}{d} \hat{u}}$.

Based on a recursive formula for finite-time ruin probabilities, one can derive another Lundberg-type bound for $\psi\left(u, x_{0}, \ldots, x_{-n+2}\right)$. Denote by $\psi_{k}\left(u, x_{0}, \ldots, x_{-n+2}\right)$ the ruin probability up to time $k$. Clearly, for $k=1,2, \ldots$, we have

$$
\begin{aligned}
& \psi_{k+1}\left(u, x_{0}, \ldots, x_{-n+2}\right)=\bar{F}_{X}\left(u+c-\sum_{i=1}^{n-1} d_{i} x_{1-i}\right) \\
& \quad+\int_{0}^{u+c-\sum_{i=1}^{n-1} d_{i} x_{1-i}} \psi_{k}\left(u+c-x-\sum_{i=1}^{n-1} d_{i} x_{1-i}, x, x_{0}, \ldots, x_{-n+3}\right) d F_{X}(x) .
\end{aligned}
$$

Hence, we have the next upper bound.

Proposition 3.3. Assume that

$$
e^{\frac{\rho}{d} \sum_{i=1}^{n-2} d_{i+1} x_{1-i}} \mathbb{E}\left[e^{\frac{\rho}{d} X\left(1+d_{1}\right)} I_{\left[X \leq u+c-\sum_{i=1}^{n-1} d_{i} x_{1-i}\right]}\right] \leq \mathbb{E}\left[e^{\rho X} I_{\left[X \leq u+c-\sum_{i=1}^{n-1} d_{i} x_{1-i}\right]}\right] .
$$

Then,

$$
\psi\left(u, x_{0}, \ldots, x_{-n+2}\right) \leq \beta e^{-\frac{\rho}{d}\left(u-\sum_{i=1}^{n-1} d_{i} x_{1-i}\right)},
$$

where

$$
\beta^{-1}=\inf _{t \geq 0} \frac{\int_{t}^{\infty} e^{\rho y} d F_{X}(y)}{e^{\frac{\rho}{d} t} \bar{F}_{X}(t)}
$$


Proof. For any $x \geq 0$, we have

$$
\begin{aligned}
\bar{F}_{X}(x) & =\left(\frac{\int_{x}^{\infty} e^{\rho y} d F_{X}(y)}{e^{\frac{\rho}{d} x} \bar{F}_{X}(x)}\right)^{-1} e^{-\frac{\rho}{d} x} \int_{x}^{\infty} e^{\rho y} d F_{X}(y) \\
& \leq \beta e^{-\frac{\rho}{d} x} \int_{x}^{\infty} e^{\rho y} d F_{X}(y) \\
& \leq \beta e^{-\frac{\rho}{d} x} \mathbb{E}\left[e^{\rho X}\right]
\end{aligned}
$$

Then,

$$
\begin{aligned}
\psi_{1}\left(u, x_{0}, \ldots, x_{-n+2}\right) & \leq \beta e^{-\frac{\rho}{d}\left(u+c-\sum_{i=1}^{n-1} d_{i} x_{1-i}\right)} \mathbb{E}\left[e^{\rho X}\right] \\
& =\beta e^{-\frac{\rho}{d}\left(u-\sum_{i=1}^{n-1} d_{i} x_{1-i}\right)} .
\end{aligned}
$$

Now, let us assume that

$$
\psi_{k}\left(u, x_{0}, \ldots, x_{-n+2}\right) \leq \beta e^{-\frac{\rho}{d}\left(u-\sum_{i=1}^{n-1} d_{i} x_{1-i}\right)} .
$$

Thus

$$
\begin{aligned}
\psi_{k}\left(u+c-x-\sum_{i=1}^{n-1} d_{i} x_{1-i},\right. & \left., x_{0}, \ldots, x_{-n+3}\right) \\
\leq & \beta e^{-\frac{\rho}{d}\left(u+c-x-\sum_{i=1}^{n-1} d_{i} x_{1-i}-d_{1} x-\sum_{i=1}^{n-2} d_{i+1} x_{1-i}\right)} \\
& =\beta e^{-\frac{\rho}{d}\left(u+c-\sum_{i=1}^{n-1} d_{i} x_{1-i}\right)} e^{\frac{\rho}{d}\left(x\left(1+d_{1}\right)+\sum_{i=1}^{n-2} d_{i+1} x_{1-i}\right)} .
\end{aligned}
$$

Furthermore,

$$
\bar{F}_{X}\left(u+c-\sum_{i=1}^{n-1} d_{i} x_{1-i}\right) \leq \beta e^{-\frac{\rho}{d}\left(u+c-\sum_{i=1}^{n-1} d_{i} x_{1-i}\right)} \int_{u+c-\sum_{i=1}^{n-1} d_{i} x_{1-i}}^{\infty} e^{\rho x} d F_{X}(x)
$$

Consequently, by (3.11), we have

$$
\begin{aligned}
& \psi_{k+1}\left(u, x_{0}, \ldots, x_{-n+2}\right) \leq \beta e^{-\frac{\rho}{d}\left(u+c-\sum_{i=1}^{n-1} d_{i} x_{1-i}\right)} \\
& \qquad\left(\int_{u+c-\sum_{i=1}^{n-1} d_{i} x_{1-i}}^{\infty} e^{\rho x} d F_{X}(x)+\int_{0}^{u+c-\sum_{i=1}^{n-1} d_{i} x_{1-i}} e^{\frac{\rho}{d}\left(x\left(1+d_{1}\right)+\sum_{i=1}^{n-2} d_{i+1} x_{1-i}\right)} d F_{X}(x)\right)
\end{aligned}
$$

By (3.12) we then get

$$
\psi_{k+1}\left(u, x_{0}, \ldots, x_{-n+2}\right) \leq \beta e^{-\frac{\rho}{d}\left(u-\sum_{i=1}^{n-1} d_{i} x_{1-i}\right)} .
$$

Letting $k \rightarrow \infty$, we obtain (3.13).

Obviously, for $n=2$, the assumption (3.12) is always fulfilled. For larger $n$, let us rewrite equation (3.12) as

$$
\begin{aligned}
& \mathbb{E}\left[e^{\frac{\rho}{d}\left(X\left(1+d_{1}\right)+d_{2} x_{0}+d_{3} x_{-1}+\cdots+d_{n-1} x_{-n+3}\right)} I_{\left[X \leq u+c-\sum_{i=1}^{n-1} d_{i} x_{1-i}\right]}\right] \\
& \leq \mathbb{E}\left[e^{\frac{\rho}{d}\left(X\left(1+d_{1}\right)+d_{2} X+d_{3} X+\cdots+d_{n-1} X\right)} I_{\left[X \leq u+c-\sum_{i=1}^{n-1} d_{i} x_{1-i}\right]}\right]
\end{aligned}
$$


It then becomes clear that for any distribution of $X$, condition (3.12) is fulfilled for at least small past claim amounts $x_{1-n+2}, x_{1-n+3}, \ldots, x_{0}$ (for instance trivially for $\left.x_{1-n+2}=x_{1-n+3}=\cdots=x_{0}=0\right)$.

The new bound (3.13) can be more precise than (3.7). For example, if $X \sim \operatorname{Exp}(\tau)$, then

$$
\beta^{-1}=\inf _{t \geq 0}\left(\frac{\tau}{\tau-\rho}\right) e^{\rho\left(1-\frac{1}{d}\right) t}=\frac{\tau}{\tau-\rho} .
$$

So, for instance, with $n=2,(3.7)$ becomes

$$
e^{-\frac{\rho}{d}\left(u-\frac{d_{1}}{\tau}\right)}
$$

while (3.13) is

$$
\left(\frac{\tau-\rho}{\tau}\right) e^{-\frac{\rho}{d}\left(u-d_{1} x_{0}\right)},
$$

which for some values of parameters $\tau, \eta$ (and hence $\rho$ ), $d_{1}$ and $x_{0}$, is smaller than (3.17).

\section{Asymptotic behavior for the ultimate ruin probabil- ity}

\subsection{The light-tailed case}

The following result makes clear that the exponential decay rate of the numerator in (3.4) provides the significant asymptotic behavior in $u$ of $\psi\left(u, x_{0}, \ldots, x_{-n+2}\right)$.

Theorem 4.1. Assume that the assumptions of Theorem 3.1 are fulfilled. Hence, given that for all $m$, the sum of the first $m$ annual losses $Z_{m}=\sum_{i=1}^{m}\left(Y_{i}-c\right)$ possesses a finite moment-generating function $\mathbb{E}\left[e^{r Z_{m}}\right]$ for $0<r<r_{0}$, where $r_{0}>\frac{\rho}{d}$, we have

$$
\lim _{u \rightarrow \infty} \frac{1}{u} \ln \psi\left(u, x_{0}, \ldots, x_{-n+2}\right)=-\frac{\rho}{d} .
$$

Proof. By the Gärtner-Ellis Theorem from large deviations (cf. Glynn and Whitt (1994), Nyrhinen (1998) or Müller and Pflug (2001)), one only has to show that:

(A1) $\kappa(r):=\lim _{m \rightarrow \infty} \frac{1}{m} \kappa_{m}(r)=\lim _{m \rightarrow \infty} \frac{1}{m} \ln \mathbb{E}\left[e^{r Z_{m}}\right]$ exists for $0<r<r_{0}$,

(A2) $\kappa\left(\frac{\rho}{d}\right)=0$ and $\kappa^{\prime}\left(\frac{\rho}{d}\right)>0$.

First of all, we prove that

$$
\lim _{m \rightarrow \infty} \frac{1}{m} \ln \mathbb{E}\left[e^{r Z_{m}}\right]=-r c+\ln \mathbb{E}\left[e^{r d X}\right] .
$$


Obviously, we have

$$
\lim _{m \rightarrow \infty} \frac{1}{m} \ln \mathbb{E}\left[e^{r Z_{m}}\right]=-r c+\lim _{m \rightarrow \infty} \frac{1}{m} \ln \mathbb{E}\left[e^{r \sum_{i=1}^{m} Y_{i}}\right] .
$$

Now

$$
\lim _{m \rightarrow \infty} \sum_{i=1}^{m} Y_{i}=d \lim _{m \rightarrow \infty} \sum_{i=1}^{m} X_{i}+\sum_{i=1}^{n-1} \sum_{k=i}^{n-1} d_{k} x_{i-k}
$$

Hence,

$$
\begin{aligned}
\lim _{m \rightarrow \infty} \frac{1}{m} \ln \mathbb{E}\left[e^{r \sum_{i=1}^{m} Y_{i}}\right] & =\lim _{m \rightarrow \infty} \frac{1}{m} \ln \mathbb{E}\left[e^{r d \sum_{i=1}^{m} X_{i}} e^{r \sum_{i=1}^{n-1} \sum_{k=i}^{n-1} d_{k} x_{i-k}}\right] \\
& =\lim _{m \rightarrow \infty} \frac{1}{m}\left(r \sum_{i=1}^{n-1} \sum_{k=i}^{n-1} d_{k} x_{i-k}\right)+\lim _{m \rightarrow \infty} \frac{1}{m} \ln \prod_{i=1}^{m} \mathbb{E}\left[e^{r d X_{i}}\right] \\
& =\lim _{m \rightarrow \infty} \frac{1}{m} \ln \mathbb{E}\left[e^{r d X}\right]^{m}=\ln \mathbb{E}\left[e^{r d X}\right] .
\end{aligned}
$$

Now, notice that $\lim _{m \rightarrow \infty} \frac{1}{m} \ln \mathbb{E}\left[e^{r Z_{m}}\right]=\ln h(r)$, where $h(r):=e^{-r c} \mathbb{E}\left[e^{r d X}\right]$. Since $h\left(\frac{\rho}{d}\right)=1$ by (3.3), we have $\kappa\left(\frac{\rho}{d}\right)=\ln h\left(\frac{\rho}{d}\right)=0$ and $\kappa^{\prime}\left(\frac{\rho}{d}\right)>0$.

Given that ruin occurs, one can also look at the path leading to ruin. Using large deviations results of Glynn and Whitt (1994), we can see that for large $u$, ruin occurs roughly at time $\left\lfloor u / \kappa^{\prime}\left(\frac{\rho}{d}\right)\right\rfloor$. Furthermore, the cumulant generating function of $Z_{\left\lfloor u / \kappa^{\prime}\left(\frac{\rho}{d}\right)\right\rfloor}$ is asymptotically changed from $\kappa_{\left\lfloor u / \kappa^{\prime}\left(\frac{\rho}{d}\right)\right\rfloor}(r)$ to

$$
\begin{aligned}
\kappa_{\left\lfloor u / \kappa^{\prime}\left(\frac{\rho}{d}\right)\right\rfloor}\left(r+\frac{\rho}{d}\right)-\kappa_{\left\lfloor u / \kappa^{\prime}\left(\frac{\rho}{d}\right)\right\rfloor}\left(\frac{\rho}{d}\right)= & -r\left\lfloor u / \kappa^{\prime}\left(\frac{\rho}{d}\right)\right\rfloor c+r \sum_{i=1}^{n-1} \sum_{k=i}^{n-1} d_{k} x_{i-k} \\
& +\left(\left\lfloor u / \kappa^{\prime}\left(\frac{\rho}{d}\right)\right\rfloor-n+1\right) \ln \frac{\mathbb{E}\left[e^{\left(r+\frac{\rho}{d}\right) d X}\right]}{\mathbb{E}\left[e^{\rho X}\right]} \\
& +\sum_{i=1}^{n-1} \ln \frac{\mathbb{E}\left[e^{\left(r+\frac{\rho}{d}\right)\left(1+\sum_{j=1}^{i-1} d_{j}\right) X}\right]}{\mathbb{E}\left[e^{\frac{\rho}{d}\left(1+\sum_{j=1}^{i-1} d_{j}\right) X}\right]} .
\end{aligned}
$$

Consequently, given that ruin occurs, on an asymptotic level the claim size distribution $X$ is exponentially tilted by the (time-dependent) factor $\rho(k), k=1,2, \ldots,\left\lfloor u / \kappa^{\prime}\left(\frac{\rho}{d}\right)\right\rfloor$, with

$$
\rho(k)=\left\{\begin{array}{ll}
\rho, & \text { for } k=1, \ldots,\left\lfloor u / \kappa^{\prime}\left(\frac{\rho}{d}\right)\right\rfloor-n+1 \\
\frac{\rho}{d}\left(1+\sum_{j=1}^{\left\lfloor u / \kappa^{\prime}\left(\frac{\rho}{d}\right)\right\rfloor-k} d_{j}\right), & \text { for } k=\left\lfloor u / \kappa^{\prime}\left(\frac{\rho}{d}\right)\right\rfloor-n+2, \ldots,\left\lfloor u / \kappa^{\prime}\left(\frac{\rho}{d}\right)\right\rfloor .
\end{array} .\right.
$$

This suggests that a sample path leading to ruin asymptotically locally (at time $k$ ) has a drift $\delta(k)$ given by

$$
\delta(k)=c-d \frac{\mathbb{E}\left[X e^{\rho(k) X}\right]}{\mathbb{E}\left[e^{\rho(k) X}\right]}<0 .
$$

Notice that the drift can be interpreted as constant during the time period $\left(1,\left\lfloor u / \kappa^{\prime}\left(\frac{\rho}{d}\right)\right\rfloor-\right.$ $n+1)$ and increasing at the end, i.e. during the time period $\left(\left\lfloor u / \kappa^{\prime}\left(\frac{\rho}{d}\right)\right\rfloor-n+2,\left\lfloor u / \kappa^{\prime}\left(\frac{\rho}{d}\right)\right\rfloor\right)$. 
However, since $u \rightarrow \infty$, this last time period is negligible compared to the first one.

Define the surplus process

$$
\tilde{U}_{k}=\tilde{u}+k c-d \sum_{i=1}^{k} X_{i},
$$

which for $\tilde{u}=\hat{u}$ corresponds to the associated non-delayed classical version of (2.2). Denote by $\widetilde{\psi}(\tilde{u})$ the corresponding ultimate ruin probability. Clearly, the Lundberg coefficient $\tilde{\rho}$ of this associated non-delayed process is equal to $\frac{\rho}{d}$. Thus, the logarithmic asymptotic behavior of $\widetilde{\psi}(\tilde{u})$ and $\psi\left(u, x_{0}, \ldots, x_{-n+2}\right)$ is of the same order. This consitutes an insensitivity property of the model. Due to the short-range dependence induced by the claim settlement, only the distribution of $d X$ is important asymptotically, and not the way to settle the claims (cf. Brémaud (2000), Yuen et al. (2005) and Ganesh and Torrisi (2006) for a similar conclusion for other delayed-claims risk models).

Remark. A large deviation estimate (consistent with (4.1)) can be obtained for the finitetime ruin probability for our risk process in the presence of IBNR claims. Indeed, let $\psi(u, M)=\operatorname{Pr}[T<M]$ denote the probability of ruin up to time horizon $M$ and define for $a>0$ the quantity $r_{a}$ as the unique solution of $\kappa^{\prime}\left(r_{a}\right)=\frac{1}{a}$. Then, invoking Nyrhinen (1998),

$$
\lim _{u \rightarrow \infty} \frac{1}{u} \ln \psi(u, a u)=-\rho_{a}
$$

with

$$
\rho_{a}= \begin{cases}r_{a}-a \kappa\left(r_{a}\right), & a<\frac{1}{\kappa^{\prime}\left(\frac{\rho}{d}\right)} \\ \frac{\rho}{d}, & a \geq \frac{1}{\kappa^{\prime}\left(\frac{\rho}{d}\right)} .\end{cases}
$$

\subsection{The heavy-tailed case}

Let us now consider subexponential claim distributions of regularly varying type. By definition, a d.f. $F$ on $\mathbb{R}^{+}$belongs to the class $R$ of regularly varying distributions $(F \in R(-\delta))$ if there exists some constant $0 \leq \delta<\infty$ and a positive slowly varying function $L(x)$ such that

$$
\bar{F}(x)=x^{-\delta} L(x) .
$$

Let $\overline{B_{0}}(x)=\frac{1}{\mu_{Z}} \int_{x}^{\infty} \overline{F_{Z}}(z) d z$, where $Z=d X-c$. If $F_{X} \in R(-\delta-1)$ for some $\delta>0$, then

$$
\lim _{u \rightarrow \infty} \frac{\psi\left(u, x_{0}, \ldots, x_{-n+2}\right)}{\overline{B_{0}}(u)}=1
$$

To show (4.9), we adapt the proof of Proposition 3.1 in Ganesh and Torrisi (2006) to our setting. 
We know by Theorem 2(B) in Veraverbeke (1977) that

$$
\lim _{\tilde{u} \rightarrow \infty} \frac{\widetilde{\psi}(\tilde{u})}{\overline{B_{0}}(\tilde{u})}=1 .
$$

Clearly, with $\tilde{u}=\hat{u}, \tilde{U}_{k} \leq U_{k}$ for all $k=1,2, \ldots$ Hence, the inequality

$$
\psi\left(u, x_{0}, \ldots, x_{-n+2}\right) \leq \widetilde{\psi}(\hat{u})
$$

holds. Consequently, we can write

$$
\lim _{u \rightarrow \infty} \sup \frac{\psi\left(u, x_{0}, \ldots, x_{-n+2}\right)}{\overline{B_{0}}(u)} \leq \lim _{u \rightarrow \infty} \frac{\widetilde{\psi}(\hat{u})}{\overline{B_{0}}(u)}=\lim _{u \rightarrow \infty} \frac{\widetilde{\psi}(\hat{u})}{\overline{B_{0}}(\hat{u})} \lim _{u \rightarrow \infty} \frac{\overline{B_{0}}(\hat{u})}{\overline{B_{0}}(u)}=\lim _{u \rightarrow \infty} \frac{\overline{B_{0}}(\hat{u})}{\overline{B_{0}}(u)}
$$

by (4.10). Since $F_{X} \in R(-\delta-1)$ (and hence $F_{Z} \in R(-\delta-1)$ ), it follows from Karamata's Theorem (see Embrechts et al. (1997)) that $B_{0} \in R(-\delta)$, which implies that

$$
\lim _{u \rightarrow \infty} \frac{\overline{B_{0}}(\hat{u})}{\overline{B_{0}}(u)}=1 \text {. }
$$

Now, notice that for $k \geq n$

$$
\sum_{i=1}^{k} Y_{i} \geq \sum_{i=1}^{k-n+1} d X_{i}
$$

This implies that

$$
\begin{aligned}
\psi\left(u, x_{0}, \ldots, x_{-n+2}\right) & =\operatorname{Pr}\left[\sum_{i=1}^{k} Y_{i}-k c>u \text { for some } k>0\right] \\
& \geq \operatorname{Pr}\left[\sum_{i=1}^{k} Y_{i}-k c>u \text { for some } k \geq n\right] \\
& \geq \operatorname{Pr}\left[\sum_{i=1}^{k-n+1} d X_{i}-k c>u \text { for some } k \geq n\right] \\
& =\operatorname{Pr}\left[\sum_{i=1}^{k-n+1} d X_{i}-(k-n-1) c>u+(n+1) c \text { for some } k \geq n\right] \\
& =\operatorname{Pr}\left[\sum_{i=1}^{k} d X_{i}-k c>u+(n+1) c \text { for some } k>0\right] \\
& =\widetilde{\psi}(u+(n+1) c) .
\end{aligned}
$$

Consequently, we get

$$
\begin{aligned}
\lim _{u \rightarrow \infty} \inf \frac{\psi\left(u, x_{0}, \ldots, x_{-n+2}\right)}{\overline{B_{0}}(u)} & \geq \lim _{u \rightarrow \infty} \frac{\widetilde{\psi}(u+(n+1) c)}{\overline{B_{0}}(u)} \\
& =\lim _{u \rightarrow \infty} \frac{\widetilde{\psi}(u+(n+1) c)}{\overline{B_{0}}(u+(n+1) c)} \lim _{u \rightarrow \infty} \frac{\overline{B_{0}}(u+(n+1) c)}{\overline{B_{0}}(u)}=1
\end{aligned}
$$


by (4.10) and $B_{0} \in R(-\delta)$.

So, as in the light-tailed claims case, the ruin probability is asymptotically insensitive to the way to settle the claims.

\section{Impact of IBNR dynamics on ultimate ruin proba- bility}

Inequality (4.11) may indicate that the IBNR dynamics decrease the risk for the insurer, as the occurred claims are paid out more evenly over time. But a "fairer" comparison in terms of the invoked initial capital is in order.

Let us return to the equivalent representation (2.3) for the annual claim amount and let $Y_{i}^{(n)}$ denote the annual claim amount for year $i$ and $\beta_{j}^{(n)}$ the proportion of $S_{i}$ paid after $j$ years (given that the number of years needed to settle the claims equals $n$ ). So, if we define $\psi^{(n)}(u)=\mathbb{E}\left[\psi\left(u, S_{0}, \ldots, S_{-n+2}\right)\right]$, we want to know whether the inequality

$$
\psi^{(n)}(u) \leq \widetilde{\psi}(u)
$$

holds true for all $u \geq 0$.

Before going on, let us briefly recall some notions on ordering (for more details, see e.g. Denuit et al. (2005)). Given two random variables $X$ and $Y, X$ precedes $Y$ in convex order (denoted by $X \leq_{\mathrm{cx}} Y$ ), if the inequality $\mathbb{E}[g(X)] \leq \mathbb{E}[g(Y)]$ holds for any convex function $g$ such that the expectations exist. Next, consider a vector $\vec{x} \in \mathbb{R}_{+}^{k}$, and its elements ranked in decreasing order $x_{(1: k)} \geq x_{(2: k)} \geq \cdots \geq x_{(k: k)}$. Given $\vec{x}, \vec{y} \in \mathbb{R}_{+}^{k}$, the vector $\vec{y}$ is said to majorize $\vec{x}$ (denoted by $\vec{x} \leq_{\text {maj }} \vec{y}$ ), if

$$
\sum_{i=1}^{j} x_{(i: k)} \leq \sum_{i=1}^{j} y_{(i: k)} \text { for } j=1,2, \ldots, k-1 \text { and } \sum_{i=1}^{k} x_{i}=\sum_{i=1}^{k} y_{i} .
$$

Proposition 5.1. For all $n=2,3, \ldots$ and $k=1,2, \ldots$, we have

$$
\sum_{i=1}^{k} Y_{i}^{(n)} \leq_{c x} \sum_{i=1}^{k} Y_{i}^{(1)}
$$

Proof. From (2.3), we get

$$
\sum_{i=1}^{k} Y_{i}^{(n)}=\sum_{i=-n+2}^{k} \gamma_{i, k}^{(n)} S_{i}
$$


with

$$
\gamma_{i, k}^{(n)}= \begin{cases}\sum_{j=-i+1}^{\min (n, k-i+1)} \beta_{j}^{(n)} & \text { if } i \leq 0 \\ 1 & \text { if } 1 \leq i \leq k-n+1 \\ \sum_{j=1}^{k+1-i} \beta_{j}^{(n)}, & \text { if } i \geq k-n+2\end{cases}
$$

For any $n \geq 2$, define the $(k+n-1)$-dimensional vectors $\vec{\phi}=\left(\overrightarrow{0}, \gamma_{1, k}^{(1)}, \gamma_{2, k}^{(1)}, \ldots, \gamma_{k, k}^{(1)}\right)$ and $\vec{\chi}=\left(\gamma_{-n+2, k}^{(n)}, \gamma_{-n+3, k}^{(n)}, \ldots, \gamma_{k, k}^{(n)}\right)$. Obviously,

$$
\vec{\chi} \leq_{\text {maj }} \vec{\phi}
$$

Indeed, $\sum_{i=1}^{k+n-1} \chi_{i}=\sum_{i=-n+2}^{k} \gamma_{i, k}^{(n)}=\sum_{i=1}^{k+n-1} \phi_{i}=\sum_{i=1}^{k} \gamma_{i, k}^{(1)}=k$. Secondly $\gamma_{i, k}^{(n)} \leq 1$ and $\gamma_{i, k}^{(1)}=1$. Consequently, by the i.i.d. property of the $S_{i}$ 's and Property 3.4 .48 of Denuit et al. (2005), we get

$$
\sum_{i=1}^{k} Y_{i}^{(1)}=\sum_{i=1}^{k+n-1} \phi_{i} S_{i-n+1} \geq_{\mathrm{cx}} \sum_{i=1}^{k+n-1} \chi_{i} S_{i-n+1}=\sum_{i=1}^{k} Y_{i}^{(n)} .
$$

This result seems to be in favor of (5.1). However, notice that in discrete-time risk models, there does not exist any result showing that the classification in the convex order sense of the aggregate claims processes implies the classification of the ruin probabilities.

Let us examine the inequality (5.1) by some numerical illustrations. Consider motor third-party liability insurance (MTPL) and pecuniary losses insurance (PL). On the basis of run-off triangles containing incremental claims for the entire Belgian market over the period 1992 - 2006 for motor TPL insurance and over the period $1997-2006$ for the pecuniary losses insurance, we get:

\begin{tabular}{|c|c|c|c|c|c|c|}
\hline \multicolumn{7}{|c|}{ MTPL insurance } \\
\hline$\beta_{1}$ & $\beta_{2}$ & $\beta_{3}$ & $\beta_{4}$ & $\beta_{5}$ & $\beta_{6}$ & $\beta_{7}$ \\
$37.79 \%$ & $26.13 \%$ & $8.50 \%$ & $5.94 \%$ & $4.78 \%$ & $3.91 \%$ & $2.96 \%$ \\
\hline$\beta_{8}$ & $\beta_{9}$ & $\beta_{10}$ & $\beta_{11}$ & $\beta_{12}$ & $\beta_{13}$ & $\beta_{14}$ \\
$2.43 \%$ & $2.03 \%$ & $1.51 \%$ & $1.44 \%$ & $1.09 \%$ & $0.89 \%$ & $0.60 \%$ \\
\hline
\end{tabular}

\begin{tabular}{|c|c|c|c|c|c|c|c|c|}
\hline \multicolumn{10}{|c|}{ PL insurance } \\
\hline$\beta_{1}$ & $\beta_{2}$ & $\beta_{3}$ & $\beta_{4}$ & $\beta_{5}$ & $\beta_{6}$ & $\beta_{7}$ & $\beta_{8}$ & $\beta_{9}$ \\
$57.46 \%$ & $37.19 \%$ & $3.60 \%$ & $1.34 \%$ & $0.25 \%$ & $0.09 \%$ & $0.04 \%$ & $0.01 \%$ & $0.02 \%$ \\
\hline
\end{tabular}

The reader interested in the methods to determine the $\beta_{j}$ is referred to Kaas et al. (2008).

Let us assume that $\mathbb{E}[S]=10$. Based on 1000000 simulations of the claims amounts $S_{i}$ for $i=-12, \ldots, 500$ for $S \sim \operatorname{Gam}\left(q_{1}, q_{2}\right)\left(\mathbb{E}\left[\operatorname{Gam}\left(q_{1}, q_{2}\right)\right]=q_{1} q_{2}\right.$ and $\mathbb{V}\left[\operatorname{Gam}\left(q_{1}, q_{2}\right)\right]=$ $\left.q_{1} q_{2}^{2}\right)$ and alternatively for $S \sim \log N(\mu, \sigma)\left(\mathbb{E}[\log N(\mu, \sigma)]=e^{\mu+\sigma^{2} / 2}\right.$ and $\mathbb{V}[\log N(\mu, \sigma)]=$ $\left.e^{2 \mu+\sigma^{2}}\left(e^{\sigma^{2}}-1\right)\right)$, we have the following numerical results for $\eta=0.1$ : 


\begin{tabular}{|c|c|c|c|c|c|c|}
\hline \multicolumn{7}{|c|}{$S \sim \operatorname{Gam}\left(q_{1}, q_{2}\right)$, with $\mathbb{E}[S]=10$} \\
\hline & & $u=0$ & $u=50$ & $u=100$ & $u=150$ & $u=200$ \\
\hline \multirow{3}{*}{$q_{1}=1$} & $\psi^{(\mathrm{MTPL})}(u)$ & 0.7035 & 0.2547 & 0.1048 & 0.0428 & 0.0175 \\
\hline & $\psi^{(\mathrm{PI}}$ & 0.7900 & 0.3094 & 0.1288 & 0.0528 & 0.0212 \\
\hline & & 0.8234 & 0.3405 & 0.1407 & 0.0578 & 0.0235 \\
\hline & & $u=0$ & $u=25$ & $u=50$ & $u=75$ & $u=100$ \\
\hline \multirow{3}{*}{$q_{1}=2$} & $\psi^{(\mathrm{MTPL})}(u)$ & 0.6213 & 0.2150 & 0.0892 & 0.0369 & 0.0154 \\
\hline & $\psi^{(\mathrm{PL})}(u)$ & 0.7363 & 0.2923 & 0.1203 & 0.0493 & 0.0199 \\
\hline & $\widetilde{\psi}(u)$ & 0.7797 & 0.3269 & 0.1360 & 0.0569 & 0.0236 \\
\hline & & $u=0$ & $u=15$ & $u=30$ & $u=45$ & $u=60$ \\
\hline \multirow{3}{*}{$q_{1}=3$} & $\psi^{(\mathrm{MTPL})}(u)$ & 0.5608 & 0.2042 & 0.0908 & 0.0411 & 0.0186 \\
\hline & $\psi^{(\mathrm{PL})}(u)$ & 0.6931 & 0.2999 & 0.1348 & 0.0607 & 0.0278 \\
\hline & $\widetilde{\psi}(u)$ & 0.7460 & 0.3442 & 0.1559 & 0.0701 & 0.0319 \\
\hline & & $u=0$ & $u=15$ & $u=30$ & $u=45$ & $u=60$ \\
\hline \multirow{3}{*}{$q_{1}=4$} & $\psi^{(\mathrm{MTPL})}(u)$ & 0.5140 & 0.1377 & 0.0474 & 0.0164 & 0.0059 \\
\hline & $\psi^{(\mathrm{PL})}(u)$ & 0.6572 & 0.2166 & 0.0744 & 0.0258 & 0.0091 \\
\hline & $\widetilde{\psi}(u)$ & 0.7182 & 0.2580 & 0.0892 & 0.0312 & 0.0110 \\
\hline & & $u=0$ & $u=10$ & $u=20$ & $u=30$ & $u=40$ \\
\hline \multirow{3}{*}{$q_{1}=5$} & $\psi^{(\mathrm{MTPL})}(u)$ & 0.4752 & 0.1504 & 0.0608 & 0.0250 & 0.0104 \\
\hline & ${\underset{\sim}{(\mathrm{PL})}(u)}^{(u)}$ & 0.6315 & 0.2475 & 0.1041 & 0.0436 & 0.0183 \\
\hline & $\widetilde{\psi}(u)$ & 0.6951 & 0.3009 & 0.1255 & 0.0521 & 0.0216 \\
\hline \multirow{4}{*}{$q_{1}=6$} & & $u=0$ & $u=10$ & $u=20$ & $u=30$ & $u=40$ \\
\hline & $\psi^{(\mathrm{MTPL})}(u)$ & 0.4399 & 0.1108 & 0.0377 & 0.0132 & 0.0046 \\
\hline & $\underset{\sim}{\psi_{\sim}^{(\mathrm{PL})}(u)}$ & 0.6055 & 0.1980 & 0.0693 & 0.0233 & 0.0080 \\
\hline & $\widetilde{\psi}(u)$ & 0.6736 & 0.2449 & 0.0853 & 0.0295 & 0.0106 \\
\hline & & $u=0$ & $u=10$ & $u=20$ & $u=30$ & $u=40$ \\
\hline & $\psi^{(\mathrm{MTPL})}(u)$ & 0.4083 & 0.0838 & 0.0236 & 0.0066 & 0.0019 \\
\hline & $\psi_{\sim}^{(\mathrm{PL})}(u)$ & 0.5861 & 0.1624 & 0.0469 & 0.0135 & 0.0041 \\
\hline & $\widetilde{\psi}(u)$ & 0.6520 & 0.1995 & 0.0583 & 0.0165 & 0.0048 \\
\hline & & $u=0$ & $u=5$ & $u=10$ & $u=15$ & $u=20$ \\
\hline \multirow{3}{*}{$=8$} & $\psi^{(\mathrm{MTPL})}(u)$ & 0.3830 & 0.1358 & 0.0638 & 0.0308 & 0.0150 \\
\hline & $\psi^{(\mathrm{PL})}(u)$ & 0.5618 & 0.2612 & 0.1283 & 0.0630 & 0.0313 \\
\hline & $\widetilde{\psi}(u)$ & 0.6385 & 0.3347 & 0.1660 & 0.0816 & 0.0404 \\
\hline \multirow{4}{*}{$q_{1}=9$} & & $u=0$ & $u=5$ & $u=10$ & $u=15$ & $u=20$ \\
\hline & $\psi^{(\mathrm{MTPL})}(u)$ & 0.3606 & 0.1138 & 0.0491 & 0.0201 & 0.0087 \\
\hline & $\psi^{(\mathrm{PL})}(u)$ & 0.5431 & 0.2276 & 0.1023 & 0.0465 & 0.0209 \\
\hline & $\widetilde{\psi}(u)$ & 0.6221 & 0.2943 & 0.1324 & 0.0613 & 0.0275 \\
\hline & & $u=0$ & $u=5$ & $u=10$ & $u=15$ & $u=20$ \\
\hline \multirow{3}{*}{$q_{1}=10$} & $\psi^{(\mathrm{MTPL})}(u)$ & 0.3390 & 0.0953 & 0.0369 & 0.0150 & 0.0061 \\
\hline & $\psi^{(\mathrm{PL})}(u)$ & 0.5243 & 0.2012 & 0.0848 & 0.0363 & 0.0159 \\
\hline & $\widetilde{\psi}(u)$ & 0.6077 & 0.2701 & 0.1118 & 0.0461 & 0.0190 \\
\hline
\end{tabular}




\begin{tabular}{|c|c|c|c|c|c|c|}
\hline \multicolumn{7}{|c|}{$S \sim \log N(\mu, \sigma)$, with $\mathbb{E}[S]=10$} \\
\hline & & $u=0$ & $u=1000$ & $u=2000$ & $u=3000$ & $u=4000$ \\
\hline \multirow{4}{*}{$\mu=0.2$} & $\psi^{(\mathrm{MTPL})}(u)$ & 0.7444 & 0.1908 & 0.0800 & 0.0400 & 0.0228 \\
\hline & $\psi^{(\mathrm{PL})}(u)$ & 0.7777 & 0.1989 & 0.0825 & 0.0411 & 0.0233 \\
\hline & $\widetilde{\psi}(u)$ & 0.7950 & 0.2022 & 0.0837 & 0.0417 & 0.0236 \\
\hline & & $u=0$ & $u=1000$ & $u=2000$ & $u=3000$ & $u=4000$ \\
\hline \multirow{4}{*}{$\mu=0.4$} & $\psi^{(\mathrm{MTPL})}(u)$ & 0.7552 & 0.1672 & 0.0619 & 0.0282 & 0.0151 \\
\hline & $\psi^{(\mathrm{PL})}(u)$ & 0.8037 & 0.1756 & 0.0641 & 0.0292 & 0.0157 \\
\hline & $\widetilde{\psi}(u)$ & 0.8067 & 0.1775 & 0.0649 & 0.0297 & 0.0159 \\
\hline & & $u=0$ & $u=1000$ & $u=2000$ & $u=3000$ & $u=4000$ \\
\hline \multirow{4}{*}{$\mu=0.6$} & $\psi^{(\mathrm{MTPL})}(u)$ & 0.7645 & 0.1397 & 0.0445 & 0.0182 & 0.0090 \\
\hline & $\psi^{(\mathrm{PL})}(u)$ & 0.7988 & 0.1464 & 0.0462 & 0.0187 & 0.0093 \\
\hline & $\widetilde{\psi}(u)$ & 0.8168 & 0.1491 & 0.0469 & 0.0191 & 0.0095 \\
\hline & & $u=0$ & $u=500$ & $u=1000$ & $u=1500$ & $u=2000$ \\
\hline \multirow{4}{*}{$\mu=0.8$} & $\psi^{(\mathrm{MTPL})}(u)$ & 0.7725 & 0.2469 & 0.1084 & 0.0526 & 0.0278 \\
\hline & $\psi^{(\mathrm{PL})}(u)$ & 0.8065 & 0.2607 & 0.1138 & 0.0549 & 0.0289 \\
\hline & $\widetilde{\psi}(u)$ & 0.8252 & 0.2665 & 0.1162 & 0.0560 & 0.0295 \\
\hline & & $u=0$ & $u=500$ & $u=1000$ & $u=1500$ & $u=2000$ \\
\hline \multirow{3}{*}{$\mu=1.0$} & $\psi^{(\mathrm{MTPL})}(u)$ & 0.7767 & 0.2037 & 0.0746 & 0.0308 & 0.0143 \\
\hline & $\psi^{(\mathrm{PL})}(u)$ & 0.8122 & 0.2166 & 0.0787 & 0.0322 & 0.0150 \\
\hline & $\widetilde{\psi}(u)$ & 0.8317 & 0.2224 & 0.0807 & 0.0329 & 0.0153 \\
\hline & & $u=0$ & $u=250$ & $u=500$ & $u=750$ & $u=1000$ \\
\hline \multirow{4}{*}{$\mu=1.2$} & $\psi^{(\mathrm{MTPL})}(u)$ & 0.7782 & 0.3060 & 0.1515 & 0.0781 & 0.0420 \\
\hline & $\psi^{(\mathrm{PL})}(u)$ & 0.8155 & 0.3291 & 0.1625 & 0.0836 & 0.0449 \\
\hline & $\widetilde{\psi}(u)$ & 0.8353 & 0.3379 & 0.1667 & 0.0858 & 0.0461 \\
\hline & & $u=0$ & $u=250$ & $u=500$ & $u=750$ & $u=1000$ \\
\hline \multirow{4}{*}{$\mu=1.4$} & $\psi^{(\mathrm{MTPL})}(u)$ & 0.7745 & 0.2388 & 0.0937 & 0.0382 & 0.0166 \\
\hline & $\psi^{(\mathrm{PL})}(u)$ & 0.8140 & 0.2587 & 0.1012 & 0.0413 & 0.0178 \\
\hline & $\widetilde{\psi}(u)$ & 0.8370 & 0.2671 & 0.1044 & 0.0427 & 0.0184 \\
\hline & & $u=0$ & $u=125$ & $u=250$ & $u=375$ & $u=500$ \\
\hline \multirow{4}{*}{$\mu=1.6$} & $\psi^{(\mathrm{MTPL})}(u)$ & 0.7638 & 0.3072 & 0.1509 & 0.0760 & 0.0387 \\
\hline & $\psi^{(\mathrm{PL})}(u)$ & 0.8081 & 0.3400 & 0.1669 & 0.0838 & 0.0428 \\
\hline & $\widetilde{\psi}(u)$ & 0.8325 & 0.3544 & 0.1738 & 0.0872 & 0.0447 \\
\hline & & $u=0$ & $u=125$ & $u=250$ & $u=375$ & $u=500$ \\
\hline \multirow{4}{*}{$\mu=1.8$} & $\psi^{(\mathrm{MTPL})}(u)$ & 0.7405 & 0.1863 & 0.0591 & 0.0193 & 0.0064 \\
\hline & $\psi^{(\mathrm{PL})}(u)$ & 0.7905 & 0.2126 & 0.0675 & 0.0220 & 0.0072 \\
\hline & $\widetilde{\psi}(u)$ & 0.8188 & 0.2246 & 0.0712 & 0.0230 & 0.0076 \\
\hline & & $u=0$ & $u=50$ & $u=100$ & $u=150$ & $u=200$ \\
\hline \multirow{3}{*}{$\mu=2.0$} & $\psi^{(\mathrm{MTPL})}(u)$ & 0.6883 & 0.2129 & 0.0817 & 0.0320 & 0.0124 \\
\hline & $\psi_{\sim}^{(\mathrm{PL})}(u)$ & 0.7517 & 0.2565 & 0.0991 & 0.0388 & 0.0151 \\
\hline & $\widetilde{\psi}(u)$ & 0.7887 & 0.2791 & 0.1072 & 0.0420 & 0.0166 \\
\hline
\end{tabular}


As expected, the inequality (5.1) is satisfied in these examples since we always have $\psi^{(\mathrm{MTPL})}(u) \leq \widetilde{\psi}(u)$ and $\psi^{(\mathrm{PL})}(u) \leq \widetilde{\psi}(u)$. However, in the heavy-tailed case the gap is less pronounced. This is in accordance with the intuition that for heavy tails ruin is the consequence of one big jump, so that the smoothing effect of the annual claim amounts induced by the IBNR dynamics is not as strong as in the light-tailed case.

\section{Conclusion}

In this paper, we have studied a discrete time risk process describing the surplus of an insurance company at the end of each calendar year. Our analysis explicitly allows for a certain delay in claim settlement, a situation commonly encountered in practice. After having derived Lundberg-type inequalities for the ultimate ruin probability, the asymptotic behavior of this probability has been studied, separately for the light-tailed case and for the heavy-tailed case. We have also compared the situations with and without delay in claim settlement, giving some quantitative results as well as numerical illustrations to what extent the insurance company can benefit from the delay in claim settlement in terms of ruin probabilities when the link factors are given. As outlined in the introduction, the analysis of this paper also has potential applications in other businesses and growth models, including the determination of health care provisions and general warranty management processes.

\section{Acknowledgements}

The authors would like to express their gratitude to anonymous referees and to the editor whose comments have been useful to revise a previous version of the present work. Michel Denuit and Julien Trufin acknowledge the financial support of the Communauté française de Belgique under contract "Projet d'Actions de Recherche Concertée" ARC 04/09-320. Hansjörg Albrecher acknowledges financial support of the Swiss National Science Foundation Project 200021-124635/1 and the Austrian Science Fund Project P18392. Michel Denuit also thanks for financial support of the Banque Nationale de Belgique under the grant "Risk measures and Economic capital".

\section{References}

[1] P. Boogaert and J. Haezendonck. Delay in claim settlement. Insurance: Mathematics and Economics, 8:321-330, 1989.

[2] P. Brémaud. An insensitivity property of Lundberg's estimate for delayed claims. Journal of Applied Probability, 37:914-917, 2000. 
[3] M. Denuit, J. Dhaene, M. Goovaerts and R. Kaas. Actuarial theory for dependent risks. John Wiley and Sons, 2005.

[4] P. Embrechts, C. Klüppelberg and T. Mikosch. Modelling extremal events. SpringerVerlag, New-York, 1997.

[5] A. Ganesh and G.L. Torrisi. A class of risk processes with delayed claims: ruin probability estimates under heavy tail conditions. Journal of Applied Probability, 43(4):916-926, 2006.

[6] A. Ganesh, C. Macci and G.L. Torrisi. A class of risk processes with reserve-dependent premium rate: sample path large deviations and importance sampling. Queueing Syst, 55:83-94, 2007.

[7] H. Gerber. Ruin theory in the linear model. Insurance: Mathematics and Economics, 1:177-184, 1982.

[8] P. W. Glynn and W. Whitt. Logarithmic asymptotics for steady-state tail probabilities in a single-server queue. Journal of Applied Probability, 31A:131-156, 1994.

[9] R. Kaas, M.J., Goovaerts, J., Dhaene and M. Denuit. Modern actuarial risk theory using $R$. Springer, New York, 2008.

[10] C. Klüppelberg and T. Mikosch. Explosive Poisson shot noise processes with applications to risk reserves. Bernoulli, 1: 125-147, 1995.

[11] C. Macci, G. Stabile and G.L. Torrisi. Lundberg parameters for non standard risk processes. Scandinavian Actuarial Journal, 417-432, 2005.

[12] C. Macci and G.L. Torrisi. Asymptotic results for perturbed risk processes with delayed claims. Insurance: Mathematics and Economics, 34(2):307-320, 2004.

[13] A. Müller and G. Pflug. Asymptotic ruin probabilities for risk processes with dependent increments. Insurance: Mathematics and Economics, 28: 381-392, 2001.

[14] H. Nyrhinen. Rough descriptions of ruin for a general class of surplus processes. Advances in Applied Probability, 30(4):1008-1026, 1998.

[15] T. Rolski, H. Schmidli, V. Schmidt and J. Teugels. Stochastic processes for insurance and finance. John Wiley and Sons, 1999.

[16] N. Veraverbeke. Asymptotic behavior of Wiener-Hopf factors of a random walk. Stochastic Processes Appl., 5(1):27-37, 1977.

[17] H.R. Waters and A. Papatriandafylou. Ruin probabilities allowing for delay in claims settlement. Insurance: Mathematics and Economics, 4: 113-122, 1985. 
[18] H. Yang. Non exponential bounds for ruin probability with interest effect included. Scandinavian Actuarial Journal, 66-79, 1998.

[19] K.C. Yuen, J. Guo and K.W. Ng. On ultimate ruin in a delayed-claims risk model. Journal of Applied Probability, 42:163-174, 2005.

\section{Appendix}

Let us consider the following dynamics for the insurer's surplus

$$
U_{k}^{(i)}=u+c \sum_{j=1}^{k}(1+i)^{j}-\sum_{j=1}^{k} Y_{j}(1+i)^{k-j} .
$$

The premiums are assumed to be paid at the beginning of each year while the claims are assumed to be paid at the end of the year. Denote by

$$
\begin{aligned}
& \psi^{(i)}\left(u, x_{0}, x_{-1}, \ldots, x_{-n+2}\right) \\
& \quad=\operatorname{Pr}\left[U_{k}^{(i)}<0 \text { for some } k \mid U_{0}^{(i)}=u, X_{0}=x_{0}, X_{-1}=x_{-1}, \ldots, X_{-n+2}=x_{-n+2}\right]
\end{aligned}
$$

the ultimate ruin probability relative to the new surplus dynamics (7.1). One can prove that if there exists a positive constant $\rho^{(i)}$ such that

$$
e^{-\frac{\rho^{(i)}}{d} c} \mathbb{E}\left[e^{\frac{\rho^{(i)}}{d} v X\left(1+\sum_{j=1}^{n-1} v^{j} d_{j}\right)}\right]=1,
$$

then we have

$$
\psi^{(i)}\left(u, x_{0}, \ldots, x_{-n+2}\right) \leq \frac{e^{-\frac{\rho^{(i)}}{d} \hat{u}^{(i)}}}{\mathbb{E}\left[e^{-\frac{\rho^{(i)}}{d} v^{T} \hat{U}_{T}^{(i)}} \mid T<\infty\right]},
$$

where $v=\frac{1}{1+i}, \hat{U}_{k}^{(i)}=U_{k}^{(i)}-\sum_{l=0}^{n-2} X_{k-l} \sum_{j=1}^{n-l-1} v^{j} d_{l+j}$, with $\hat{u}^{(i)}=\hat{U}_{0}^{(i)}$ and $T=\inf \{k$ : $\left.U_{k}^{(i)}<0\right\}$. Indeed, we have

$$
\begin{aligned}
\mathbb{E}\left[e^{-\frac{\rho^{(i)}}{d} v^{k} \hat{U}_{k}^{(i)}} \mid \Phi_{k-1}\right] & =\mathbb{E}\left[e^{-\frac{\rho^{(i)}}{d} v^{k}\left(U_{k-1}^{(i)}(1+i)+c(1+i)-Y_{k}-\sum_{l=0}^{n-2} X_{k-l} \sum_{j=1}^{n-l-1} v^{j} d_{l+j}\right)} \mid \Phi_{k-1}\right] \\
& =\mathbb{E}\left[e^{-\frac{\rho^{(i)}}{d} v^{k-1}\left(U_{k-1}^{(i)}+c-v\left[Y_{k}+\sum_{l=0}^{n-2} X_{k-l} \sum_{j=1}^{n-l-1} v^{j} d_{l+j}\right]\right)} \mid \Phi_{k-1}\right],
\end{aligned}
$$

where $\Phi_{k}$ is the sigma-algebra generated by $\left\{X_{i}, i=1,2, \ldots, k\right\}$. Now

$$
v\left[Y_{k}+\sum_{l=0}^{n-2} X_{k-l} \sum_{j=1}^{n-l-1} v^{j} d_{l+j}\right]=X_{k}\left(v+\sum_{j=1}^{n-1} v^{j+1} d_{j}\right)+\sum_{l=0}^{n-2} X_{k-1-l} \sum_{j=1}^{n-l-1} v^{j} d_{l+j} .
$$

Thus, we get

$$
\begin{aligned}
\mathbb{E}\left[e^{-\frac{\rho^{(i)}}{d} v^{k} \hat{U}_{k}^{(i)}} \mid \Phi_{k-1}\right]= & \mathbb{E}\left[e^{-\frac{\rho^{(i)}}{d} v^{k-1}\left(c-v X\left(1+\sum_{j=1}^{n-1} v^{j} d_{j}\right)\right)}\right] \\
& \mathbb{E}\left[e^{-\frac{\rho^{(i)}}{d} v^{k-1}\left(U_{k-1}^{(i)}-\sum_{l=0}^{n-2} X_{k-1-l} \sum_{j=1}^{n-l-1} v^{j} d_{l+j}\right)} \mid \Phi_{k-1}\right] \\
= & \mathbb{E}\left[e^{-\frac{\rho^{(i)}}{d} v^{k-1}\left(c-v X\left(1+\sum_{j=1}^{n-1} v^{j} d_{j}\right)\right)}\right] e^{-\frac{\rho^{(i)}}{d} v^{k-1}} \hat{U}_{k-1}^{(i)}
\end{aligned}
$$


Furthermore, as $i \geq 0$, it suffices to note (cf. Yang (1998)) that

$$
\begin{aligned}
\mathbb{E}\left[e^{-\frac{\rho^{(i)}}{d} v^{k-1}\left(c-v X\left(1+\sum_{j=1}^{n-1} v^{j} d_{j}\right)\right)}\right] & =\mathbb{E}\left[e^{\left(-\frac{\rho^{(i)}}{d}\left(c-v X\left(1+\sum_{j=1}^{n-1} v^{j} d_{j}\right)\right) v^{k-1}\right.}\right] \\
& \leq \mathbb{E}\left[e^{-\frac{\rho^{(i)}}{d}\left(c-v X\left(1+\sum_{j=1}^{n-1} v^{j} d_{j}\right)\right)}\right]^{v^{k-1}}
\end{aligned}
$$

by Jensen's inequality. Consequently, $\left\{e^{-\frac{\rho^{(i)}}{d} v^{k} \hat{U}_{k}^{(i)}}, k \geq 0\right\}$ is a supermartingale, since

$$
\mathbb{E}\left[e^{-\frac{\rho^{(i)}}{d}\left(c-v X\left(1+\sum_{j=1}^{n-1} v^{j} d_{j}\right)\right)}\right]=1
$$

by assumption. In view of the final arguments of the proof of Theorem 3.1, the inequality (7.3) follows. 